\title{
Impaired Body Surface Electrode Contact Reduces Accuracy of Non-Invasive Electrocardiographic Imaging
}

\author{
Laura R. Bear ${ }^{1,2,3}$, Jeanne van der Waal ${ }^{4}$, Thom F. Oostendorp ${ }^{5}$, Rémi Dubois ${ }^{1,2,3}$ \\ ${ }^{1}$ Electrophysiology and Heart Modelling Institute (IHU LIRYC), Bordeaux, France \\ ${ }^{2}$ CRCTB Inserm U1045, Bordeaux, France \\ ${ }^{3}$ Université de Bordeaux, CRCTB Inserm U1045, Bordeaux, France \\ ${ }^{4}$ Amsterdam UMC, University of Amsterdam, Clinical and Experimental Cardiology, Amsterdam, \\ the Netherlands \\ ${ }^{5}$ Donders Institute for Brain, Cognition and Behaviour, Radboud University Medical Centre, \\ Nijmegen, the Netherlands
}

\begin{abstract}
This study examines the impact of torso electrode contact on potential (MFS) and activation (EDL) based inverse methods. Inverse reconstructions were computed for simulated pacing data before and after reduction of a single torso potential's amplitude. Potential reduction severely impacted MFS reconstruction accuracy creating small areas of inverted electrograms and large activation errors near the electrode affected. The impact was more subtle for EDL, keeping the general activation pattern but creating artificial regions of slowed conduction.
\end{abstract}

\section{Introduction}

Non-invasive electrocardiographic imaging of cardiac electrical activity from body surface potentials (BSP) is a promising clinical tool for electroanatomic mapping of arrhythmogenic substrates [1,2]. Current systems typically record BSPs using disposable adhesive electrode arrays in the form of strips or vests with upwards of 250 electrodes [1,2]. This allows for redundancy, ensuring all information projected from the heart is captured and signals which are considered unusable can be discarded to improve inverse reconstructions [3].

The large number of electrodes also increases the chance of poor contact of some electrodes with the skin surface. While those not in contact can be easily identified due to the lack of signal, those in partial contact will likely produce a normal ECG waveform but with a reduced signal amplitude, particularly once contact is less than $20 \mathrm{~cm}^{2}$ [4].

While the impact of baseline wander and high frequency noise on inverse reconstructions has been studied [5], to date there has been no investigation of the impact of poor electrode contact. The aim of this study was to rectify this by determining the effect of reducing BSP amplitudes on potential and activation-based inverse solutions.

\section{Methods}

\subsection{Data}

Simulated data was obtained through the EDGAR repository (http://edgar.sci.utah.edu/), provided by the Karlsruhe Institute of Technology [6]. This data includes 8 paced ventricular beats simulated on a human patient model using a cellular automaton method. Extracellular potentials were extracted on an epi-endocardial and a pericardial heart surface. BSPs were forward-calculated using the finite element method. White Gaussian noise (30dB) was added to BSPs.

\subsection{Inverse Methods}

The inverse problem was solved using a potential- and an activation-based approach. 
Potential-based 'MFS' approach

Pericardial potentials were reconstructed using the methods described in [7]. That is, the method of fundamental solutions is used to solve the boundary value problem using zeroorder Tikhonov regularization and CRESO criterion to find the regularization parameter. Activation times (AT) were computed by fitting a global activation field to electrogram delays [8].

\section{Activation-based 'EDL' approach}

Activation times were reconstructed using the methods described in detail in [9]. In brief, an equivalent dipole later (EDL) was defined as the underlying source model. The dipole layer strength is determined by endo- and epicardial activation times. The boundary element method is used to compute BSPs generated by the EDL source assuming homogeneous conductivity within the torso. An initial estimate is computed using the fastest route algorithm, subsequently iterative optimization is used to improve the fit between forward-computed and recorded BSPs.

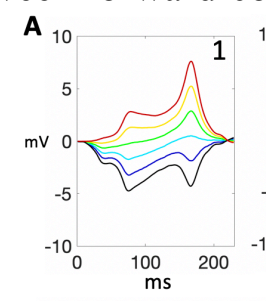

B

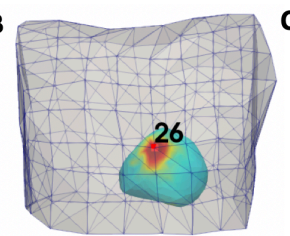

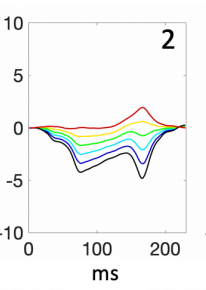

$2 \quad 10$
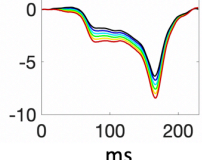

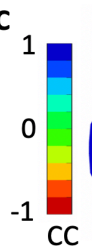

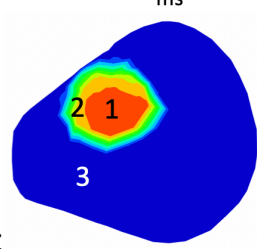

Fig 1A) MFS reconstructed electrograms with B) BSP 26 reduced $0 \%$ (black), $20 \%$ (blue), $30 \%$ (cyan), 60\% (green), 80\% (yellow) and $100 \%$ (red). C) CC map between black and red electrograms.

\subsection{Evaluation Metrics}

To simulate a single electrode with poor contact, a simple model was used of reducing the individual BSP signal amplitudes by $20 \%$, to $100 \%$. Inverse reconstructions were computed using MFS and EDL algorithms. This was performed for all 163 BSPs individually. 'Gold standard' electrograms and ATs were defined as reconstructions from BSP without amplitude reduction $(0 \%)$. Electrograms were compared using Pearson's correlation (CC), and ATs using the absolute error (ATE). For global comparisons, the $2.5 \%$ and $97.5 \%$ percentile of $\mathrm{CC}$ and ATE over the heart was calculated and defined as $\mathrm{CC}_{\min }$ and $\mathrm{ATE}_{\max }$ for each inverse.
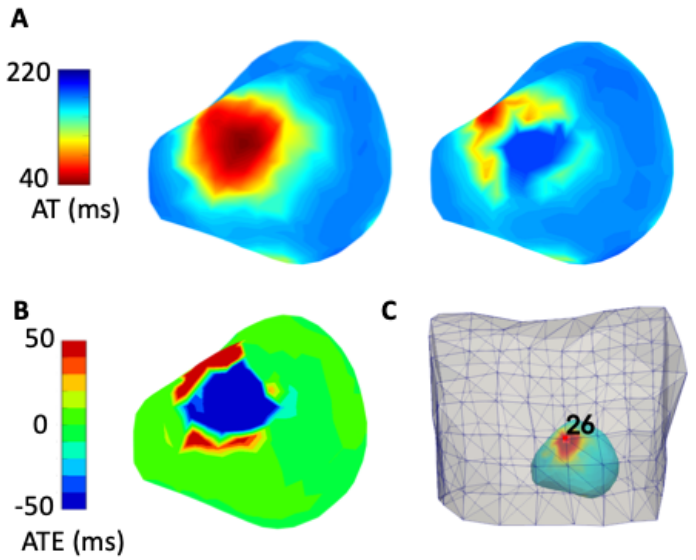

Fig 2A) MFS AT maps for 'gold standard' (left) and with BSP 26 reduced $100 \%$ (right). B) AT error between the two maps and C) position of the heart in relation to electrode 26 .

\section{Results}

\subsection{Electrogram and AT reconstruction}

Fig 1A present three 'gold standard' MFS electrograms (black) alongside those after electrode 26 (Fig 1B) was reduced $20 \%$ to $100 \%$ (blue to red). Electrogram locations are noted in the CC map (Fig 1C) between 'gold standard' electrograms and those with BSP 26 reduced to $0 \%$. As seen with electrogram 3 , the majority of electrograms kept the same morphology $(\mathrm{CC}=1)$ despite reducing $\mathrm{BSP}$ amplitude. In a small area, electrogram amplitudes were reduced in parallel with the BSP (e.g. electrograms 1 and 2). By $60 \%$ reduction (green), the electrograms invert and $\mathrm{CC}$ becomes negative.

Fig 2A presents the 'gold standard' MFS activation maps (left) for this RV anterior 
pacing sequence alongside that after BSP 26 was reduced $100 \%$ (right), one of the worst-case scenarios for the MFS method. These demonstrate the reversal of electrograms results in a distortion of the early activation region by late activation. The ATE map in Fig 2B shows the change in ATs in this area are substantial $(>50 \mathrm{~ms})$. The area of the epicardial surface most affected corresponds to the closest region to the BSP with reduced amplitude, as seen in Fig 2C showing the position of the heart with respect to BSP 26.

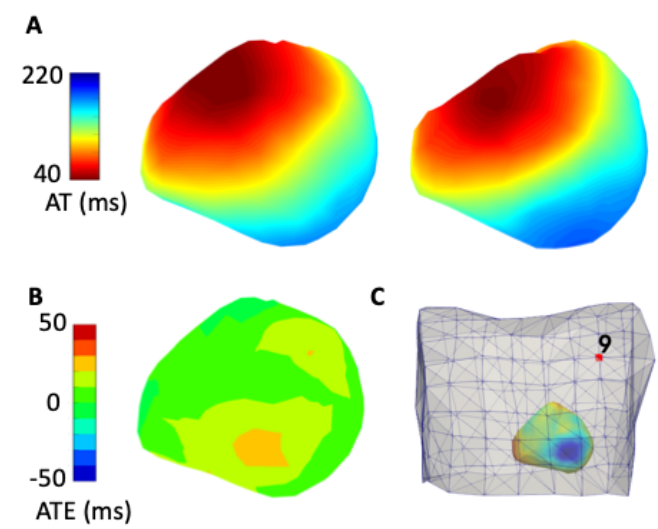

Fig 3A) EDL AT maps for 'gold standard' (left) and with BSP 9 reduced 100\% (right). B) AT error between the two maps and $\mathrm{C}$ ) position of the heart in relation to electrode 9.

Fig 3A presents a 'gold standard' EDL activation map (left) and that after BSP 9 was reduced $100 \%$ (right), one of the worst-case scenarios for the EDL method. Unlike with the MFS method, the global pattern of activation is maintained after amplitude reduction, although closer inspection reveals a region of conduction slowing near the RV apex and LV lateral wall. This is seen in Fig 3B with AT errors of 20-30 $\mathrm{ms}$ in these areas.

Fig $4 \mathrm{~A}$ presents the (top) minimum $\mathrm{CC}_{\min }$ and (bottom) the maximum region of the heart (\%) with $\mathrm{CC}<50 \%$ across all BSPs leads at different levels of amplitude reduction. Fig 4B and C present the (top) maximum $\mathrm{ATE}_{\max }$ and (bottom) the maximum region of the heart (\%) with ATE $>40 \mathrm{~ms}$ for all BSPs for the MFS and EDL methods respectively. These plots demonstrate that for the MFS method, substantial changes in electrogram morphology and ATE arise once BSP amplitude are reduced by $40 \%$, with electrogram inversion $(\mathrm{CC}<0 \%)$ occurring below 60\%. Furthermore, the impact on ATs is larger than the impact on electrograms, with a greater $\%$ of the heart affected.

For the EDL method, changes in ATs are seen more rapidly (20\% reduction) than the MFS, though ATEs are lower than for the MFS and $\%$ of the heart affected with large ATEs $(>40 \mathrm{~ms})$ is minimal ( $<5 \%$ of the heart).
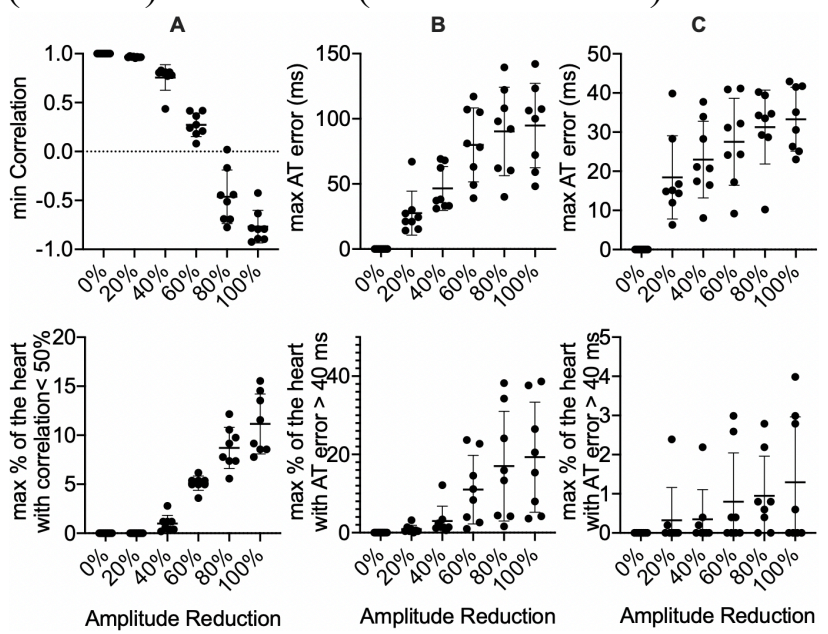

Fig 4. Comparison of A) MFS electrograms and B) activation maps, and C) EDL activation maps.

\subsection{Torso Electrodes}

Fig 5 presents the $\mathrm{CC}_{\min }$ and $\mathrm{ATE}_{\max }$ for $60 \%$ reduction of each BSP for an RV lateral and LV anterior pacing sequences for the MFS method (A and B) and the EDL method (C). For the MFS, BSPs closer to the heart have a stronger impact on $\mathrm{CC}$, and are in the same location regardless of activation sequence. The same BSPs also cause larger ATE. However, BSPs that cause only minor electrogram changes can also create large error in ATs. This occurs in the presence of electrograms with two downstrokes, where even a slight change in morphology can cause ATs to switch from one to the next, creating large errors. 
For the EDL method, while there are also only a subset of BSPs that have a major impact on the reconstruction, the position of these torso electrodes is dependent on the activation sequence. This is seen with central chest electrodes impacting accuracy of the RV lateral sequence while side electrodes most impacting the LV anterior sequence.

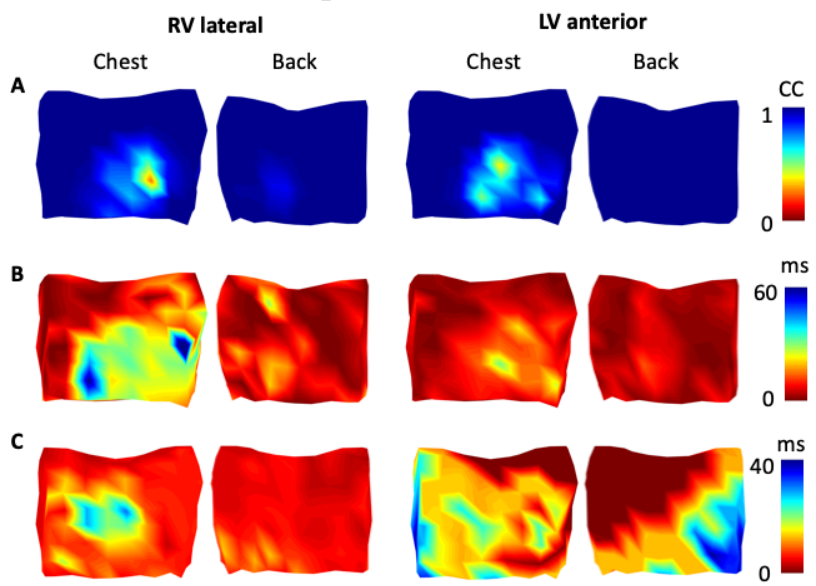

Fig 5A) $\mathrm{CC}_{\min }$ and B) $\mathrm{ATE}_{\max }$ for MFS and C) EDL reconstructions after $60 \%$ reduction.

\section{Discussion}

This study examines the impact of torso electrode contact on potential and activation.

For potential-based methods, poor electrode contact over a large proportion of the torso does not severely affect inverse reconstructions in terms of electrogram morphology or AT error. However, for electrodes closer to the heart, reconstructions can be substantially altered if amplitudes are reduced to $60 \%$ or more of their original amplitude, including reversal of electrogram morphology and large errors in AT detection in localized regions of the heart. This is an important concern for female patients, where breast morphology can result in poor electrode contact directly over the heart when using fixed arrays such as strips or vests. The benefit of such drastic localized changes in inverse solutions and the geometric dependence of BSP to the region of change means that such errors should be easy to identify and the abnormal BSP rectified.
Activation-based method are more robust to electrode contact errors than potential-based methods, with changes in reconstructions being more subtle: keeping the general activation pattern but creating artificial regions of slowed conduction. Like with potential-based methods, only a subset of torso electrodes has this impact. However, this subset and the region of cardiac tissue affected is dependent on the cardiac sequence, not the geometry. This combination of subtle changes and geometric independence means that on the rare occasion these errors do occur they will be harder to identify.

This study was limited to the evaluation of individual BSP amplitude reduction. While such isolated contact issues may occur, it is more likely for small groups of neighboring electrodes to suffer such problems. Further evaluation in this area is needed.

\section{Acknowledgments}

This work was supported by the French National Research Agency (ANR-10-IAHU04-LIRYC), and the Leducq Fondation (16CVD02).

\section{References}

1. Haissaguerre M, et al. Driver domains in persistent atrial fibrillation. Circulation. 2014;130:530-8.

2. Potyagaylo D, et al. ECG adapted fastest route algorithm to localize the ectopic excitation origin in CRT patients. Front Physiol. 2019;10:1-13.

3. Bear LR, et al. Accuracy of lead removal vs linear interpolation in non-invasive electrocardiographic imaging (ECGI). Comput Cardiol Conf (CinC), 2015. 2015. p. 941-4. 4. Fernandes MS, et al. Flexible PDMS-based dry electrodes for electro-optic acquisition of ECG signals in wearable devices. IEEE EMBC. 2010;3503-6.

5. Bear LR, et al. Effects of ECG signal processing on the inverse problem of electrocardiography. Compt Cardiol. 2018. 6. Schulze WHW, et al. A simulation dataset for ECG imaging of paced beats with models for transmural, endo- and epicardial and pericardial source imaging. ECG Imaging. 2015;1-6.

7. Bear LR, et al. Optical imaging of ventricular action potentials in a torso tank: A new platform for non-invasive electrocardiographic imaging validation. Front Physiol. 2019;10:1-11.

8. Duchateau J, et al. Spatially Coherent Activation Maps for Electrocardiographic Imaging. IEEE TBME. 2016;64:1-8. 9. Van Dam PM, et al. Non-invasive imaging of cardiac activation and recovery. Ann Biomed Eng. 2009;37:1739-56.

Dr. Laura R Bear

IHU-Liryc, Avenue Haut Leveque, Pessac, France laura.bear@ihu-liryc.fr 